\title{
Homocystinuria due to a novel mutation in MTHFR gene: a rare cause of non-immune hydrops fetalis
}

\begin{abstract}
Background: Homocystinuria is a common inborn error of metabolism usually presenting postnatally with Marfanoid features and thrombotic events. It is often caused by mutations in the $C B S$ gene and rarely by $M T H F R$ gene defect.

Case characteristics: A multigravida whose previous three neonates expired due to refractory myoclonic seizures presented with Non-immune hydrops fetalis at 32 weeks of gestation. Prenatal diagnosis with sequencing studies on amniotic fluid revealed a homozygous novel missense mutation in MTHFR gene. Neonate had Marfanoid features and microcephaly and investigations revealed elevated levels of Homocystine. Early intervention with Pyridoxine and supportive care with multi-vitamin therapy possibly averted the onset of Myoclonic seizures and death in the newborn.
\end{abstract}

Conclusion: Homocystinuria can be a rare cause of non-immune hydrops fetalis and prenatal diagnosis can help initiate immediate postnatal therapy to prevent a possible neonatal death.

Keywords: Hydrops, Neonate, Homocystinuria, MTHFR deficiency, Myoclonic seizures.
Volume 9 Issue I - 2018

\author{
Mamatha Gowda,' Vasanthan Tanigasalam, ${ }^{2}$ \\ Monica Thiagarajan, ${ }^{3}$ Nishad Plakkal, ${ }^{4}$ Vishnu \\ Bhat $^{5}$ \\ 'Assistant professor, Department of Obstetrics and Gynecology, \\ Jawaharlal Institute of Postgraduate Medical Education and \\ Research (JIPMER), India \\ ${ }^{2}$ Senior resident, Department of Neonatology, Jawaharlal \\ Institute of Postgraduate Medical Education and Research \\ (JIPMER), India \\ 3unior Resident, Department of Obstetrics and Gynecology, \\ Jawaharlal Institute of Postgraduate Medical Education and \\ Research (JIPMER), India 4Assistant professor, Department \\ of Neonatology, Jawaharlal Institute of Postgraduate Medical \\ Education and Research (JIPMER), India \\ ${ }^{4}$ Senior professor \& Head, Department of Neonatology, \\ Jawaharlal Institute of Postgraduate Medical Education and \\ Research (JIPMER), India
}

\begin{abstract}
Correspondence: Dr. Mamatha Gowda (MG), Medical Geneticist, Assistant professor, Department of Obstetrics and Gynecology, JIPMER, Pondicherry-605 006, India, Tel 9488|277|6,Email drmamathagowda@gmail.com
\end{abstract}

Received: July 24, 2017 | Published: February 15, 2018

\section{Introduction}

Homocystinuria is an inborn error of metabolism commonly resulting from mutations in the Cystathione beta synthetase (CBS) gene [OMIM \#236200] and rarely due to mutations in the MTHFR gene [\# 236250]. ${ }^{1-3}$ The clinical spectrum varies from asymptomatic adults to neonatal deaths in severely affected cases. The condition has mostly been described postnatally with Marfanoid features, ocular defects, thrombotic events, microcephaly, seizures and developmental delay. We report the first case of prenatal diagnosis of Homocystinuria due to a novel homozygous missense mutation in MTHFR gene presenting as microcephaly, arachnodactyly, pleural effusion and fetal ascites.

\section{Case report}

A non-consanguineously married fourth gravida presented with history of previous three neonatal deaths due to intractable myoclonic seizures. Screening anomaly scan done in second trimester was normal. A follow up ultrasound at 32 weeks of gestation showed fetal ascites (Figure 1A), gross right sided pleural effusion (Figure 1B) and hepatosplenomegaly. The biparietal diameter and head circumference were lagging by two weeks suggesting microcephaly. The great toe appeared broad and the other toes and fingers were slender and long (Figure 1C). Amniocentesis was done and sample analysed by limited exome sequencing study. A novel missense variation, c.121C $>\mathrm{T}$; p.Arg41Trp, in exon 1 of the MTHFR gene (chr1:11863053; G>A;) resulting in the substitution of Tryptophan for Arginine at codon 41 (p.Arg41Trp; ENST00000376592) was detected in homozygous state.

A male baby weighing $2.5 \mathrm{~kg}$ was born by vaginal delivery at term. At birth, the neonate was asymptomatic and had ascites (Figure 2A) without pleural effusion and edema of subcutaneous tissues
There was microcephaly, high arched palate, epigastric hernia, arachnodactyly, joint contractures, pectus excavatum, pes cavus and long arm span suggesting marfanoid habitus on examination (Figure 2B). Serum homocysteine level was elevated at $65 \mathrm{micromol} /$ liter and methionine was normal. The clinical presentation was consistent with homocystinuria, implying the pathogenicity of MTHFR gene variant. In view of previous neonatal deaths and elevated serum homocysteine levels, the neonate was treated with multivitamin supplements. The newborn had no seizures and ascites resolved and the baby is asymptomatic after 3 months of follow up.

\section{Discussion}

Homocystinuria is an autosomal recessive inborn error of metabolism. Classic homocystinuria is caused homozygous or compound heterozygous mutations in Cystathionine b-synthetase gene and rarely by mutations in the MTHFR gene. Affected individuals display a variable spectrum of presentation, the most common being involvement of ocular system with myopia or anterior segment dislocation of the lens, vascular thrombosis and skeletal abnormalities. Less commonly intellectual disability, failure to thrive, seizures and megaloblastic anemia can be the presenting feature. ${ }^{5}$ There are only a few reports of MTHFR deficiency homocystinuria in the literature and none reporting prenatal diagnosis to the best of our knowledge.

This is the first case describing the phenotype of MTHFR deficiency related homocystinuria in fetal life. The fetus had microcephaly, broad great toes, arachnodactyly and nonimmune hydrops with pleural effusion and ascites. The pathophysiology of fluid collection is enigmatic but hyperhomocysteinemia causing capillary endothelial vasomotor dysregulation and capillary leak can be considered. ${ }^{6}$ 

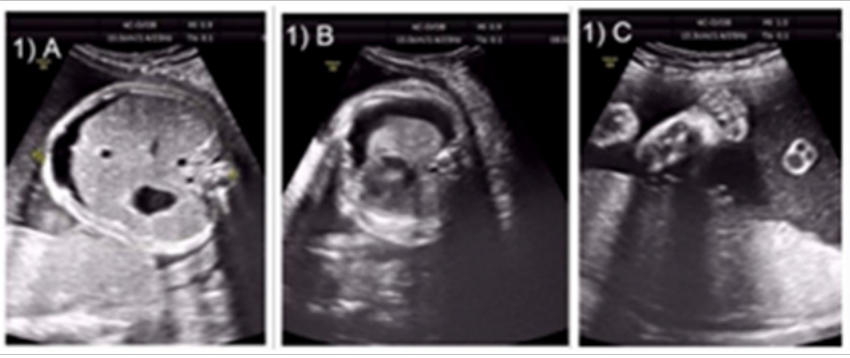

Figure I Antenatal ultrasound showing.

a. Fetal ascites

b. Right sided pleural effusion

c. Broad great toe and long toes.
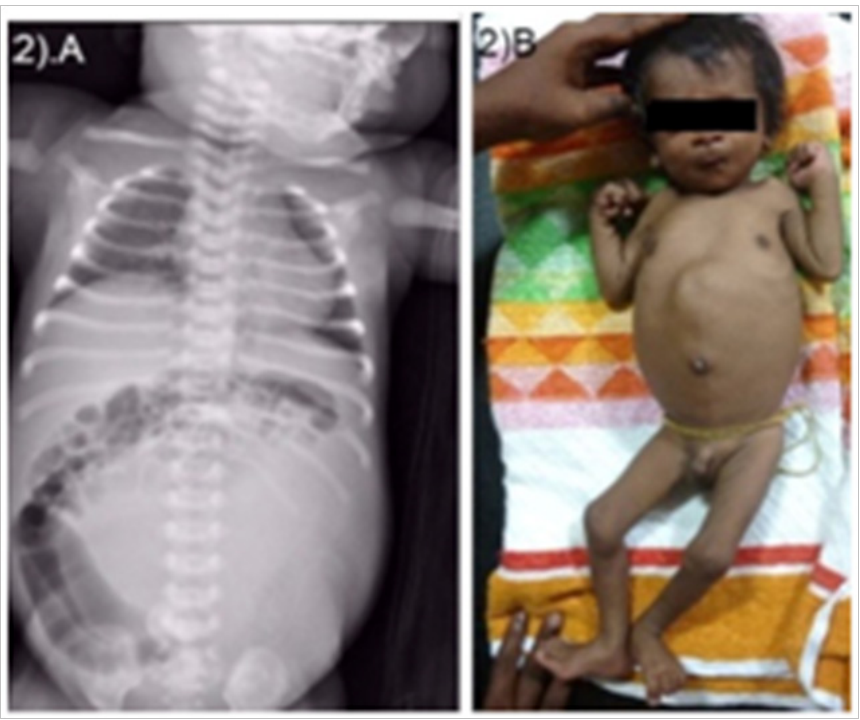

Figure 2 Postnatal images.

a. Skiagram showing ascites.

b. Phenotype showing microcephaly, arachnodactyly, joint contractures, pes cavus, pectus excavatum, epigastric hernia and marfanoid habitus (length=arm span).

In the present case, there is a history of previous three sibling's death due to refractory myoclonic seizures in the neonatal period. The etiology of those neonatal deaths is unknown, but in light of the present case, the probable cause of refractory seizures and death might have been neonatal homocystinuria. Thus the need for universal newborn screening against the preventable causes of neonatal deaths gains momentum. However, one pitfall of routine newborn screening which incorporates only methionine or homocysteine is that other causes of homocystinuria such as due to MTHFR deficiency and cobalamin defects may not be detected as the methionine level in these disorders is reduced or normal. Hence both homocysteine and methionine analysis in primary newborn screening methods can overcome this problem. ${ }^{7}$ The neonate had high homocysteine and normal methionine levels, thus differentiating it from other common causes of homocystinuria. Molecular genetic tests can be very valuable in confirming the underlying defect to facilitate prenatal diagnosis in future pregnancies.
The newborn was treated with pyridoxine, methylcobalamin and leucovorin. The treatment of homocystinuria due to MTHFR deficiency is different from the other forms. Since there is a methylation defect in the folic acid pathway, these neonates are treated with methylated form of oral vitamin B12 and folinic acid. ${ }^{8}$ The other FDA approved drug is betaine. Betaine is associated with positive outcome in severe MTHFR deficiency but the availability of the drug and the cost is the main limitation. ${ }^{9}$ These neonates are at high risk of lens dislocation, developmental delays, neuropsychiatric problems and premature atherosclerosis. Hence there is a need to plan a multidisciplinary follow up and treatment.

\section{Conclusion}

Neonatal homocystinuria is one of the preventable causes for developmental delay and neuropsychiatric problems. Homocystinuria can be a rare cause of non-immune hydrops fetalis and prenatal diagnosis can help initiate immediate postnatal therapy to prevent a possible neonatal death.

\section{Acknowledgements}

None.

\section{Conflicts of interest}

None.

\section{References}

1. Cassandra L Kniffin. Homocystinuria due to Cystathionine betasynthetase deficiency. OMIM. Johns Hopkins University, Baltimore, USA. 2010.

2. Cassandra L Kniffin. Homocystinuria due to deficiency of $\mathrm{N}(5,10)$ Methylene-tetrahydrofolate-reductase activity. OMIM. Johns Hopkins University, Baltimore, USA. 2014.

3. Dean L. Methylenetetrahydrofolate Reductase Deficiency. In: Pratt V, McLeod H, Dean L, Malheiro A \& Rubinstein W (Eds.), Medical Genetics Summaries, National Center for Biotechnology Information, Bethesda, USA. 2012.

4. De Franchis R, Sperandeo MP, Sebastio G, et al. Clinical aspects of cystathionine beta-synthase deficiency: how wide is the spectrum? The Italian Collaborative Study Group on Homocystinuria. Eur J Pediatr. 1998;157 Suppl2:S67-S70.

5. Sacharow SJ, Picker JD, Levy HL. Homocystinuria Caused by Cystathionine Beta-Synthase Deficiency. In: Pagon RA, Adam MP, Ardinger HH, Wallace SE \& Amemiya A (Eds.), Gene Reviews, University of Washington, Seattle, USA. 2004.

6. Dayal S, Lentz SR. Murine models of hyperhomocysteinemia and their vascular phenotypes. Arterioscler Thromb Vasc Biol. 2008;28(9):15961605 .

7. Snyderman SE, Sansaricq C. Newborn screening for homocystinuria. Early Hum Dev. 1997;48(1-2):203-207.

8. Huemer M, Diodato D, Schwahn B, et al. Guidelines fordiagnosis and management of the cobalamin-related remethylation disorders cblC, cblD, cblE, cblF, cblG, cblJ and MTHFR deficiency. $J$ Inherit Metab Dis. 2017;40(1):21-48.

9. Huemer M, Kožich V, Rinaldo P, et al. Newborn screening for homocystinurias and methylation disorders: systematic review and proposed guidelines. $J$ Inherit Metab Dis. 2015;38(6):1007-1019. 